\title{
Automated Analysis of Eye-Tracking Data for the Evaluation of Driver Information Systems According to ISO/TS 15007-2:2001
}

\author{
Christian Lange, Martin Wohlfarter, and Heiner Bubb \\ Lehrstuhl für Ergonomie, Technische Universiät München \\ Bolzmannstrasse 15, 85747 Garching \\ \{lange, $\mathrm{m}$.wohlfarter, bubb\} Ife.mw. tum. de
}

\begin{abstract}
First of all, the most important content of the ISO/TS 15007-2:2001 standard for performing eye tracking experiments will be described. The following text includes a detailed description of how eye/gaze experiments using the Dikablis eye tracking system are conducted according to the above mentioned standard and how recorded statistical evaluations can be automated and visualized.
\end{abstract}

Keywords: ISO/TS 15007-2:2001, Eye tracking, Driver Assistance Systems, Driver Information Systems.

\section{Introduction}

Adhering to the guidelines of the European Statement of Principles ESoP, the need for good and less distracting design of driver information systems will grow enormously. The future will yield the driver information systems which will hinder or distract the driver from the driving task being performed as least as possible. The advantage of the standardized experimental conduction lies in the improvements delivered by progressive experimental comparability, error reduced and faster processing. The duration of the analysis of uplifted data is therefore enormously reduced. Subsequently, the standard conduction of gaze experiments using the Dikablis Toolkit can be proved to comply with the ISO/TS 15007-2:2001 standard.

\section{Toolkits for Standardized Experimentation and Testing}

The Dikablis software package supports the standards required by eye tracking experimentation. In figure 1 the interference between Dikablis and the ISO/TS 150072:2001 standard regarding test planning, calculations and analysis of eye tracking data is illustrated.

Dikablis will be deployed in order to evaluate the tracking of the gaze direction and eye and head movements of the test subject. The Dikablis Toolkit consists of a Recording Software, an Analysis Software for post-processing of the eye-tracking data and manual data analysis and D-LAB for completely automated data analysis. 


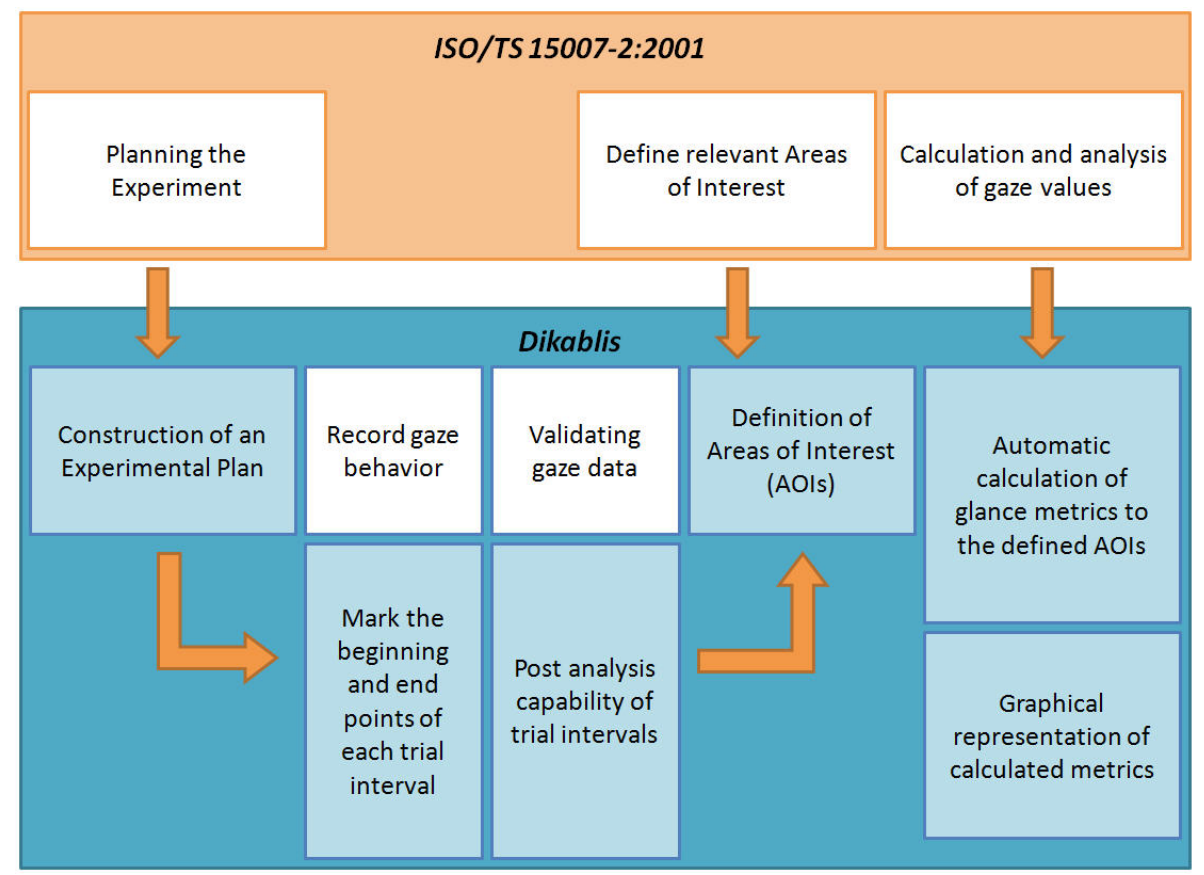

Fig. 1. Workflow schema for standardized testing and experimentation of Dikablis and ISO/TS 15007-2:2001 interferences/differences

With the Recording Software the gaze behavior of the test subject can precisely be recorded. The test subject wears the Dikablis Head Unit, on which two cameras are installed. One camera is directed towards the test subject's eye and is used to recognize the subject's gaze behavior (pupil movements). The other camera is directed straight on in front of the subject and monitors the subject's environment. By processing both of these pieces of video data, the gaze direction of the test subject can almost precisely be determined.

Offline re-calibration and post-processing of the eye-detection can be made with the Dikablis Analysis Software using the recorded data even after the recording software has finished recording. These possibilities to adjust and post-process guarantee clean and always analyzable test results under any circumstances.

The D-LAB module contains a package for conducting experiments, from test planning and defining of special Areas of Interest (AOI) visible within the gaze region, to the automatic calculation of glance durations and graphical presentation of these results.

Successively, the proceedings to standardized testing and experimentation will sbe described subsequently. Herein the capital and lower case letters are always related to figure 1's demonstrated procedural (workflow) plan and the relationships between the small angled boxes located within the figure. 


\subsection{Construction of an Experimental Plan}

The compliancy to test partitioning presented by ISO/TS 15007-2:2001 into ,experimental condition“, „task“, and ,subtask" can be defined into one test plan with DLAB (see figure 2 left). This way a test can be represented in the form of intertwined and nested intervals in which:

- „experimental condition“ an entire Experiment is unfolded (Ex. driving on country roads);

- "task" defines the interaction between a determined system presented within the Experiment (ex. Operation of Navigation Systems);

- A specification of a "task" as "subtask" will be considered. (Ex. Operation of a navigation system via a touch screen display).

D-LAB offers the possibility to additionally define „subsubtasks“ as the fourth layer, ex. in order to mark the appearance of critical events presented within the experiment (ex. sharp breaking situations) or automatic analysis of the display screen (ex. an individual input screen within the navigation system, for example inputting a destination address).
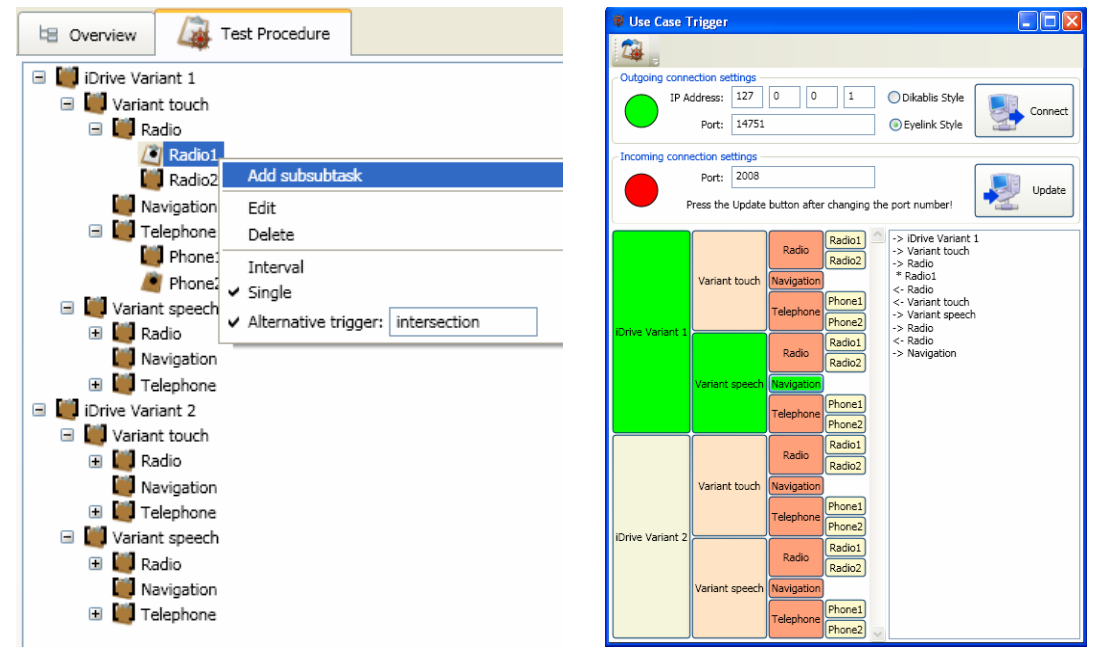

Fig. 2. Left: constructing a test sequence; Right: Automatically created block shifting diagram/interface for online test segment sequence marking

\subsection{Record Gaze Behavior and Mark the Beginning and End Points of Each Trial Interval}

Using one of the D-Lab integrated applications one can automatically create a shifting block diagram/interface using the trial definitions, which can be seen on the right side of figure 2. Each shifting block represents a test segment. By pressing a block, a network event is created, which marks the start or ending of a task interval directly in the Recording Software. The functionality of the Dikablis recording software is described 
in written detail in Lange et al., 2006a und Lange et al., 2006b. These events mark the beginning respectively the end of a trial segment and are synchronously saved to the calculated gaze data. The events can also be started from another data recorder such as a driving simulator.

\subsection{Validating Gaze Data and Trial Intervals}

The Dikablis analysis software is firm in validating the gaze data after a trial and in optimizing. In preparation, the gaze data if necessary is calibrated after the trial so as to optimize offline pupil recognition (see Lange et al., 2006a and Lange et al. 2006b). Further processing in D-LAB follows after the optional jointing is successful. The first move consists of testing whether all trial intervals would be marked correctly. The marked trial intervals located under the gaze player window synchronized to the playtime line shown on the user interface layer (see figure 3). D-LAB also offers a testing function which automatically identifies inconsistencies within the trial segment. The segment can be manually adjusted or changed, tasks can be added or deleted. Figure 3 shows the D-LAB interface for management of a trial interval.

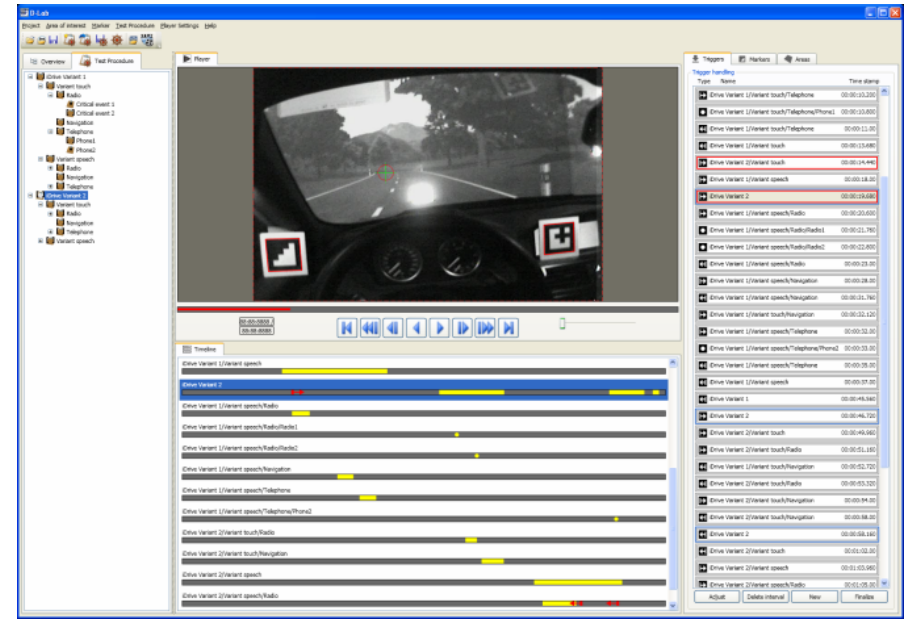

Fig. 3. Validation and post-processing of a trial segment in D-LAB

\subsection{Definition of Areas of Interest}

The areas of interest used in the calculation of glance durations in relevant gaze regions (ex. the navigation system display, the street view, the rear view mirror, the left angle mirror, the dashboard, etc.) as required by the ISO/TS 15007-2:2001 are defined by using D-LAB's functionality of marking specific AOIs. Thus in D-LAB a random number of AOIs can be defined in the form of a polyline and labeled with names. Figure 4 demonstrates the Head-Up Display and Dashboard as defined AOIs. 


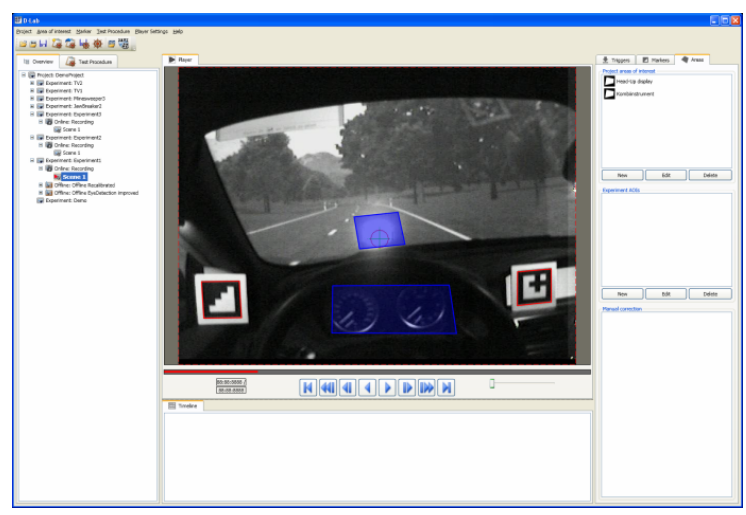

Fig. 4. Defining selected AOIs within the gaze region such as the Head-Up Display and the Dashboard

\subsection{Automatic Calculation of Glance Metrics to the Defined AOIs}

In order to automatically calculate glance duration and glance frequency, the pupil of the test subject as well as his/her head position in relation to the test environment (relative to the defined AOIs) must be recognized. The determination of the head position is carried out with the help of the so called marker(s) (see the quadratic black and white object in figure 4), which describe the environment reference. These markers are found using image processing in the photo of the field camera and used in the head position calculation of the test subject.

For every defined and established AOI, using the D-LAB function „Calculate Gaze Durations" it is calculated when the test subject glanced at an AOI and when their glance was away from a certain AOI. The result of this calculation is displayed analogously to the graphical representation of the trial intervals located under the gaze film player window synchronous to the playtime line. Hence, the operator can impose calculation corrections at any time simply by testing and manually correcting if necessary. The gaze specific values from the ISO/TS 15007-2 standard can be calculated using the automated determination of glance durations via the defined AOIs. Thus the operator can input the values which need be calculated for the following gaze experiment in the form of an "Analysis Series“. Therefore the specific values pertaining to specific tasks and AOIs to be calculated must be defined. The following values are available to choose from:

- Total Glance Time

- Glance Frequency

- Time off road-scene-ahead

- Total glance time as a percentage

- Fixation probabilities

- Link value probabilities

- Maximum Glance Duration

- Mean Glance Duration 
D-Lab hereupon calculates the inputted values and saves the results in the form of text file. These automated value calculations can be conducted for all defined AOIs, all terms of the experiment plan as well as for all gaze metrics. The text file is built concurrently so that it can be opened for further statistical analysis using the SPSS statistics program without indirection.

\subsection{Graphical Representation of Calculated Metrics}

Next to the internal calculation of values, D-LAB additionally offers a trial result(s) graphical representation capability. For this purpose several graphical diagrams are displayed which support the interpretation of the result(s). There are two examples in figure 5 that exemplify this. To the top one can find the progressive course of glance duration on defined AOIs for a single subtask presented by four independent test subjects. To the bottom the course of the users mean glance fixation duration in a critical situation is shown in order to allow conclusions on the basis of mental strain.

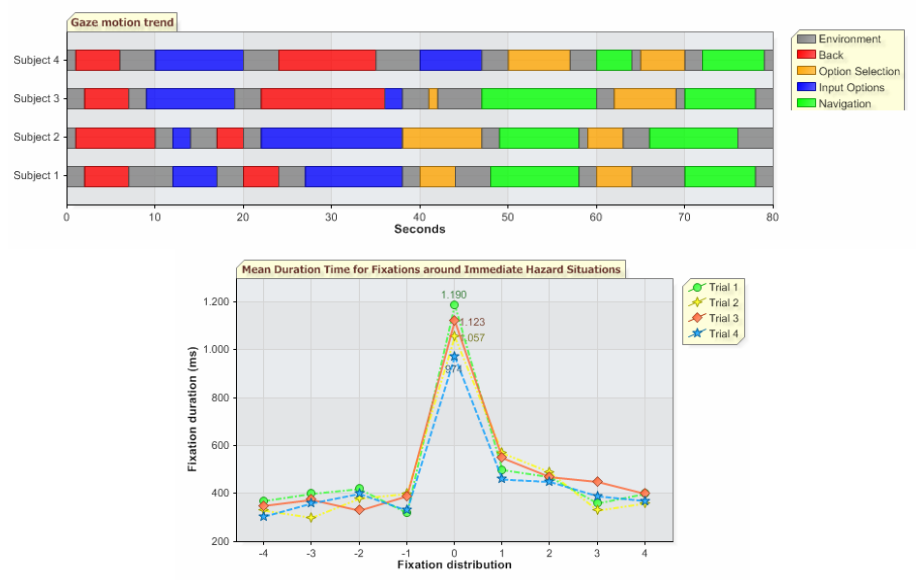

Fig. 5. Top: Glance duration on the defined AOIs on all trial experiments. Bottom: User glance fixation duration in critical situations.

\section{References}

1. ISO/TS 15007-2:2001: Road vehicles - Measurement of driver visual behavior with respect to transport information and control systems - Part 2: Equipment and procedures

2. Lange, C., Yoo, J.-W., Wohlfarter, M., Bubb, H.: Dikablis - Operation mode and evaluation of the human-machine interaction. In: Spring Conference of Ergonomics Society of Korea, Seoul, May 12 (2006)

3. Lange, C., Wohlfarter, M., Bubb, H.: Dikablis - engineering and application area. In: Proceedings IEA 2006 16th World Congress on Ergonomics, Maastricht the Netherlands (2006) 